\title{
NON DESTRUCTIVE TESTING USING TEMPORAL PHASE EVALUATION IN SPECKLE INTERFEROMETRY
}

\author{
C. Joenathan*, B. Franze, P. Haible, and H. J. Tiziani \\ Universitaet Stuttgart, Institut fuer Technische Optik, \\ Pfaffenwaldring 9, 70569 Stuttgart, \\ Germany
}

Keywords: Interferometry, Speckle Phenomena, Nondestructive testing

\begin{abstract}
A novel nondestructive testing (NDT) method where measurement of large object deformations using temporal evolution of the speckles in speckle interferometry is reported. Basic principle of the method is continuous object deformation is introduced and the fluctuations in the phase of the speckle as intensity modulation is recorded. Acquiring a large number of frames of the object motion the phase data for the whole object deformation is then retrieved by Fourier-transformation technique. The method is capable of measuring deformations of over 100 microns for in-plane and out-of-plane with speckle interferometry and over $500 \mu \mathrm{m}$ for speckle shearing interferometry. We discuss the NDT results obtained with the three methods and make some relative comparisons of each of them.
\end{abstract}

\section{Introduction:}

Speckle interferometry has found numerous NDT applications because the defect appears as a deviation of the fringes in a rather predictable fringe pattern. Electronic processing of speckle patterns has enhanced the usability of speckle methods by generating the possibility of real-time analysis with high repeatability [1-4]. In speckle interferometry because of its high sensitivity too many fringes are obtained thus making defect detection difficult. On a rule of thumb, 30 fringes or less in a 512 x 512 pixel array allow for easy identification of the defect. Consequently the upper limit on the object deformation is usually of the order of $10 \mu \mathrm{m}$. To reduce sensitivity and allow large deformations on objects oblique incidence and observation [5] and longer wavelength lasers [6] have been reported. However, these alternatives have inherent problems such as in oblique incidence the size of the prism generates an upper limit on the object size and with larger wavelengths the CCD camera becomes more expensive with lower resolution and relatively hard to operate.

The purpose of this paper is to report our study on some new methods that can be used to extract the whole object deformation using Fourier transformation methods of the speckle pattern recorded in a sequence as the object is being deformed. A large number of frames of the object motion is recorded thereby generating a temporal modulation of the intensity. By analyzing this signal, the object deformation ranging from few microns to few hundreds of microns can be determined. The range of deformation measurement is dependent on the instrumentation and the correlation between the speckle patterns. In the Fourier transformation method used in this paper, the signal is first transformed, and one side of the spectrum is filtered using a band pass filter. The filtered spectrum is then inverse transformed to obtain the phase fluctuation that is then unwrapped to get the total phase at a given point. Therefore, in principle linear deformation of the object is not necessary. The basic principle of the temporal Fourier transform analysis of the sequence of speckle pattern is the similar to the spatial domain Fourier-transform technique for fringe pattern analysis [7]. This idea was used for extracting out-of -plane [8], in-plane [9], and slope [10] information about the object deformation. Further the Fourier-transform technique has been used for measuring absolute step height and object shapes as well $[11,12]$. A short description of each method to measure outof-plane, in-plane and slope are presented. Nondestructive testing results for a metallic plate with a crack along with comparison and limitations are also outlined. 


\section{Principle of the methods:}

In all the methods the interference between object and reference beam forming a complex interference pattern is recorded on a CCD camera. The equation for intensity (after simplification of the interference equation) at an image point of the object at the sensor plane can be expressed as

$$
\begin{aligned}
\mathrm{I}(\mathrm{x}, \mathrm{y}, \mathrm{t})= & \mathrm{I}_{\mathrm{o}}(\mathrm{x}, \mathrm{y}) \\
& \{1+\mathrm{V} \cos [\Phi \mathrm{o}(\mathrm{x}, \mathrm{y})+\Phi(\mathrm{x}, \mathrm{y}, \mathrm{t})]\}
\end{aligned}
$$

Where $I_{0}(x, y)$ is the bias intensity of the speckle pattern, $\mathrm{V}$ is the visibility of the speckle modulation, $\Phi_{0}(\mathrm{x}, \mathrm{y})$ is the random phase, and $\Phi(\mathrm{x}, \mathrm{y}, \mathrm{t})$ is the phase introduced due to out-ofplane or in-plane or the slope of the object deformation. The relationship for $\Phi(\mathrm{x}, \mathrm{y}, \mathrm{t})$ depending on the setup used is

$$
\begin{aligned}
& \Phi(\mathrm{x}, \mathrm{y}, \mathrm{t})=\frac{4 \pi \mathrm{Z}(\mathrm{x}, \mathrm{y}, \mathrm{t})}{\lambda}[\text { out }- \text { of }- \text { plane }] \\
& \Phi(\mathrm{x}, \mathrm{y}, \mathrm{t})=\frac{4 \pi \mathrm{U}(\mathrm{x}, \mathrm{y}, \mathrm{t}), \mathrm{V}(\mathrm{x}, \mathrm{y}, \mathrm{t})}{\lambda \sin \theta}[\text { in - plane }] \\
& \Phi(\mathrm{x}, \mathrm{y}, \mathrm{t})=\frac{4 \pi[(\partial \mathrm{Z}(\mathrm{x}, \mathrm{y}, \mathrm{t}) / \partial \mathrm{x}) \Delta \mathrm{x}]}{\lambda}[\text { slope }]
\end{aligned}
$$

Where $\theta$ is the angle of illumination of the setup for in-plane displacement, $U$ and $\mathrm{V}$ are the inplane displacement components along the $\mathrm{x}$ and $\mathrm{y}$ directions respectively, $\Delta \mathrm{x}$ and $\Delta \mathrm{y}$ are the amount of shear along the $\mathrm{x}$ and $\mathrm{y}$ directions respectively, and for the out-of-plane sensitivity setup the angle of illumination of the object is zero. Let us consider one point on the image of the object and as the object is deformed the intensity fluctuation is recorded as a function of time. The median temporal frequency of the signal observed at a given point of the image of the object during $t$ seconds of recording is

$$
\mathrm{f}_{\text {med }}(\mathrm{x}, \mathrm{y}, \mathrm{t})=\frac{\Phi(\mathrm{x}, \mathrm{y}, \mathrm{t})}{2 \pi \pi \mathrm{t}}
$$

Thus the median temporal frequency of the signal observed is dependent on the object deformation or its slope. A series of frames of the speckle pattern is recorded as the object is being displaced. Each frame is then a record of the speckles and their intensity at that instant of time. For each pixel we observe its variation over time providing the pixel history that is the temporal modulation of the speckle intensity. By taking the Fourier-transform with respect to the time variable of each pixel history (Fourier Transform of equation 1 after further simplification) we obtain

$$
\mathrm{F}\{\mathrm{I}(\mathrm{x}, \mathrm{y}, \mathrm{t})\}=\widetilde{\sim}+\widetilde{\sim}+\mathrm{B}\left(\mathrm{f}-\mathrm{f}_{\text {med }}\right)+\widetilde{\sim}\left(-\mathrm{f}-\mathrm{f}_{\text {med }}\right)
$$

where

$$
\begin{aligned}
& \mathrm{A}=\mathrm{F}\{\mathrm{Io}(\mathrm{x}, \mathrm{y})\}, \\
& \text { B }\left(\mathrm{f}-\mathrm{f}_{\text {med }}\right)= \\
& \mathrm{F}\left\{\frac{\operatorname{Io}(\mathrm{x}, \mathrm{y})}{2} \mathrm{~V} \exp \left[\mathrm{i}\left(\Phi_{\mathrm{o}}(\mathrm{x}, \mathrm{y})+\Phi(\mathrm{x}, \mathrm{y}, \mathrm{t})\right)\right]\right\}, \\
& \widetilde{\mathrm{C}}\left(-\mathrm{f}-\mathrm{f}_{\text {med }}\right)= \\
& \mathrm{F}\left\{\frac{\mathrm{Io}(\mathrm{x}, \mathrm{y})}{2} \mathrm{~V} \exp [-\mathrm{i}(\Phi \mathrm{o}(\mathrm{x}, \mathrm{y})+\Phi(\mathrm{x}, \mathrm{y}, \mathrm{t}))]\right\},
\end{aligned}
$$

and $\mathrm{F}$ stands for Fourier transform. Equation 4 reveals that two bands are obtained on either side of a central peak in the transform plane. The carrier frequency generated due to the object displacement should be large enough to separate the side bands from the central peak. Upon isolation of one of the side bands with an appropriate band pass filter, an inverse Fourier transform is applied to the filtered spectrum giving rise to a complex term as

$$
\begin{aligned}
& \mathrm{F}\left\{\widetilde{\mathrm{B}}\left(\mathrm{f}-\mathrm{f}_{\text {med }}\right)\right\}= \\
& \mathrm{C}_{1} \frac{\operatorname{Io}(\mathrm{x}, \mathrm{y})}{2} \mathrm{~V} \exp [\mathrm{i}(\Phi \mathrm{o}(\mathrm{x}, \mathrm{y})+\Phi(\mathrm{x}, \mathrm{y}, \mathrm{t}))]
\end{aligned}
$$

Where $\mathrm{C}_{1}$ is a constant introduced by the transformation. From the angle of the complex values of the resultant transform, the phase information is extracted. The constant phase from the speckle at a point can be eliminated by observing the phase at $t=0$. This procedure of obtaining the phase does not demand that the displacement be linear. The phase is then unwrapped as in phase shifting interferometry 
and thereby a 3-D map of the time dependent phase of the object deformation can be generated. From the 3-D data, the beginning and the end phase of the object deformation can be obtained. In the present method of analysis, the instantaneous angular frequency or the linear velocity generated during deformation of the object point is determined. From these extrapolations the 3-D plot of the object deformation is extracted.

It should be noted that points on the object with zero displacement cannot be resolved. Also for points where 10 cycles or less modulation is generated a resolvable separation of the peaks in the spectrum is not obtained. Therefore, these points of the image do not have any data. This resolution is dependent on the band-pass filter used to separate one of the side order bands. To overcome this problem a constant displacement or slope is introduced based on the sensitivity of the method. Constant slope is introduced by tilting the object in the case of shearing system. This modification introduces a reference temporal frequency signal (frequency off-set) is produced which modifies equation (1) to

$$
\begin{aligned}
& \mathrm{I}(\mathrm{x}, \mathrm{y}, \mathrm{t})=\mathrm{I}_{\mathrm{o}}(\mathrm{x}, \mathrm{y}) \\
& \quad\left\{1+\mathrm{V} \cos \left[\Phi_{\mathrm{O}}(\mathrm{x}, \mathrm{y})+\Phi(\text { constan } \mathrm{t})+\Phi(\mathrm{x}, \mathrm{y}, \mathrm{t})\right]\right\}
\end{aligned}
$$

Where the phase factor $\Phi$ (constant) contains the reference frequency off set. Consequently with no object displacement there is a reference frequency. The upper limit on the displacement or slope that can be measured is set by the maximum number of cycles that can be recorded in the total number of frames. This theoretical upper limit is set by the Nyquist criterion. This limit is

$$
\begin{aligned}
& \mathrm{Z}(\mathrm{x}, \mathrm{y})=\mathrm{N}_{\mathrm{nq}} \frac{\lambda}{2} \\
& \mathrm{U}(\mathrm{x}, \mathrm{y}), \mathrm{V}(\mathrm{x}, \mathrm{y})=\mathrm{N}_{\mathrm{nq}} \frac{\lambda}{2 \sin \theta} \\
& \frac{\partial \mathrm{Z}(\mathrm{x}, \mathrm{y})}{\partial \mathrm{x}} \Delta \mathrm{x}, \frac{\partial \mathrm{Z}(\mathrm{x}, \mathrm{y})}{\partial \mathrm{y}} \Delta \mathrm{y}=\mathrm{N}_{\mathrm{nq}} \frac{\lambda}{2}
\end{aligned}
$$

Where $\mathrm{N}_{\mathrm{nq}}$ is the Nyquist frequency and in our set up is 512 cycles. Another factor is that speckle decorrelation reduces the modulation that is observed and for large deformation the modulation gradually reduces and then reappears if the object is further deformed or displaced beyond the longitudinal speckle size because a new speckle is formed. To increase the range of deformation with better accuracy (a) for out-ofplane deformation the longitudinal speckle size has to he increased and (b) for in-plane deformation the lateral speckle size has to be increased.

\section{Out-of-plane displacement:}

The schematics of the out-of-plane sensitive temporal Fourier-transform speckle interferometer for NDT is shown in Fig. 1. The configuration is similar to that of the Michelson interferometer, where one of the mirrors is replaced with the diffuse object that is being tested. A telecentric system is used to image the object onto the sensor of a high speed Charge Coupled Device (CCD) camera. In addition, the telecentric system insures the propagation axis of the beam from the mirror and the object to be collinear. It is very well known that the angle between the object and the reference beam should be such that the fringes modulating the individual speckles must be resolved by the CCD camera.

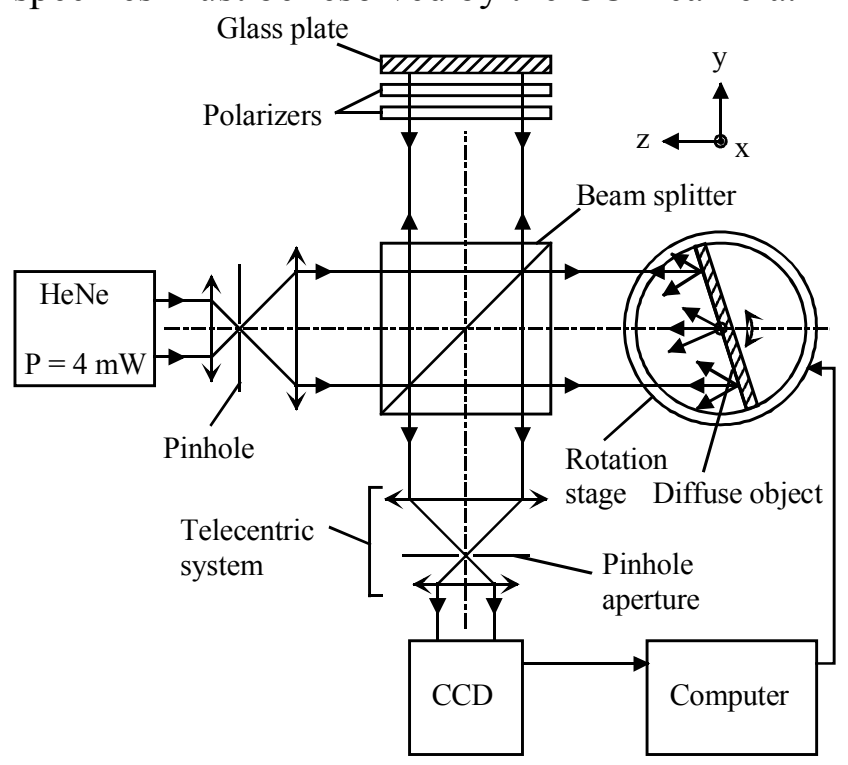

Fig. 1: Schematic of the experimental arrangement of the temporal Fourier-transform speckle interferometric method for measuring out-of-plane deformation. 


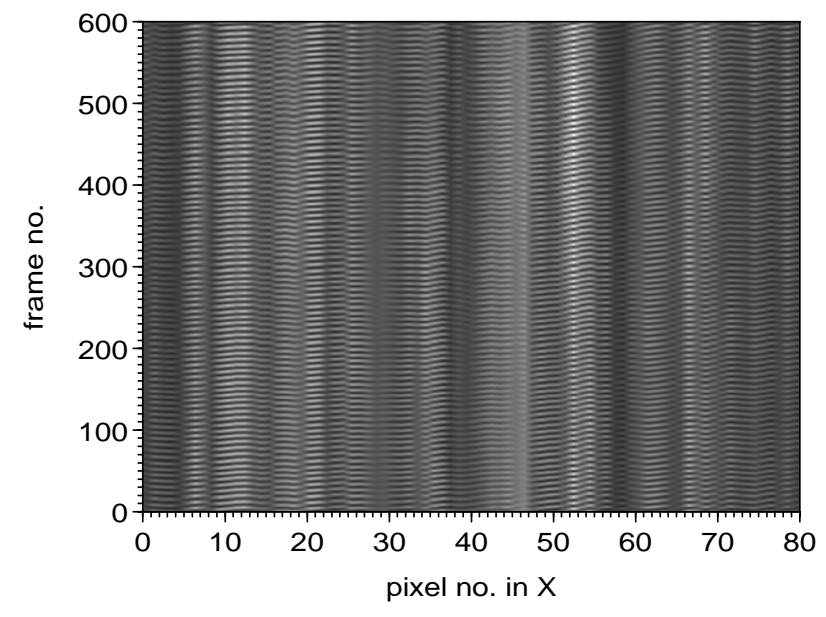

(a)

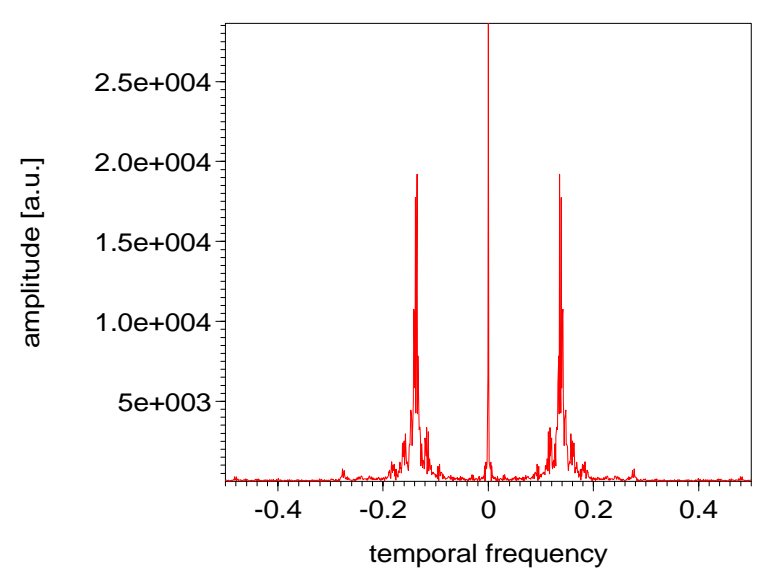

(c)

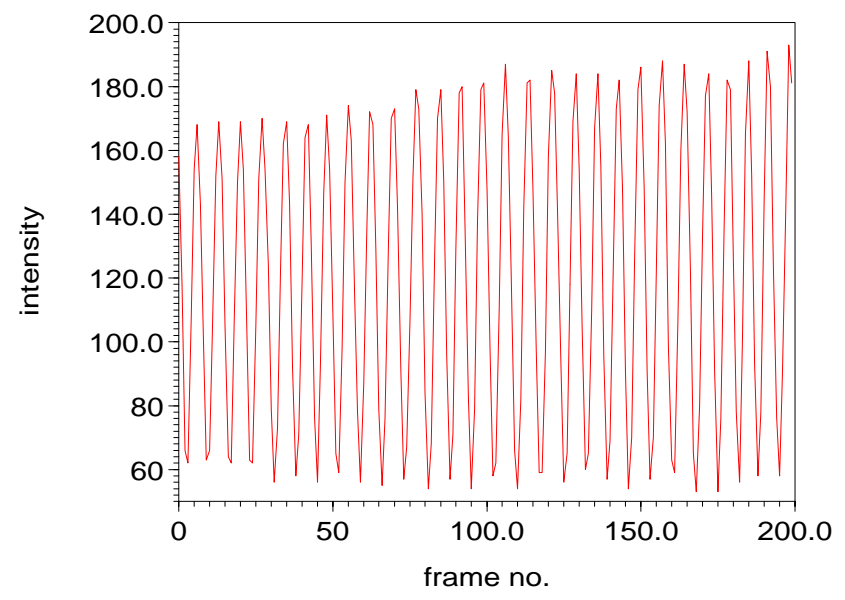

(b)

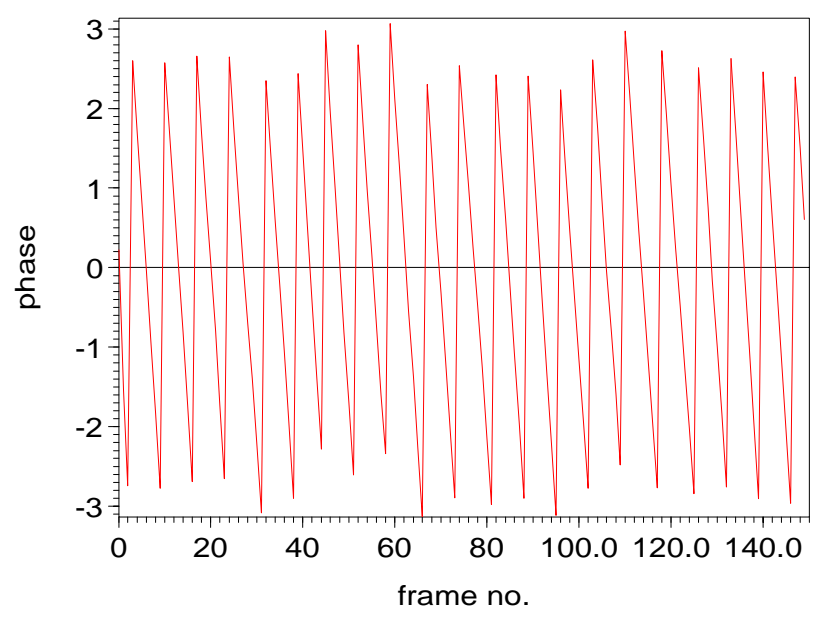

(d)

Fig. 2. Experimental results with tilting of an aluminum plate. a) Shows the slice of one horizontal line of pixel from each frame stacked together. Only 600 of the 1024 frames are shown to clearly depict the speckle modulation and the correlation. b) The intensity fluctuation of one speckle as a function of time. (c) The Fourier-transformation of the temporal signal shown in (b). (d) The filtered spectrum is again Fourier transformed. The phase values are then obtained from the complex term. The phase fluctuates between $-\pi$ and $+\pi$ and only few cycles are shown for clarity.

The basic principle of the method of extracting information here is demonstrated using the out-of-plane sensitive interferometer. A plate was rotated about the y-axis and a high speed CCD camera was used to acquire 360 frames in one second. The camera was clocked down from its normal 820 frames per second to maintain higher sensitivity. A He-Ne laser of 5 $\mathrm{mW}$ power was used in our experiments. Acquisition of the 1024 frames as well as the rotation of the stage was synchronized. Figure 2 (a) shows one horizontal slice from each frame stacked with the time along the vertical axis depicting that the speckles remain correlated over the whole deformation. In order to show the speckle intensity modulation with time not all the 1024 slices are shown in the figure. A plot of the signal modulation at a pixel is shown in Fig. 2 (b) and only 200 of the 1024 frames are shown here. The corresponding FFT (with the 1024 frames) of the signal is shown in Fig. 2 (c). One of the side spectrum is then filtered using a band pass filter and inverse Fourier transformed. The phase information is then obtained from the complex term. Through this procedure we obtain the phase fluctuations between $-\pi$ and $+\pi$ and is shown in Fig. 2 (d). Unwrapping the phase, that is by adding $2 \pi$ phase values at every discontinuity, gives the total phase of the signal and thereby the total displacement of the object. 
Intermediate displacements can therefore also be extracted.

A plate clamped along its edges and loaded at the center was used to demonstrate the potential of the present method of analysis for NDT applications. The plastic sheet of $1.0 \mathrm{~mm}$ thickness and diameter of $65 \mathrm{~mm}$ with a clear aperture of $60 \mathrm{~mm}$ was clamped almost uniformly along its edges. The object was then point loaded from the back by a step up motor operated linear translation stage via a dial gauge where the speed of translation and the distance of translation was synchronized with the data acquisition. The distance of translation was determined so that not more than 512 cycles were obtained during data acquisition of three seconds. The technique was found to be robust and immune to external disturbances. Figure 3 (a) shows the 3-D plot of the deformation of the object in a $11 \times 7 \mathrm{~mm}^{2}$ area of the center of the object. The center of the object was deformed by $126 \mu \mathrm{m}$. The contour plot of the deformation is shown in Fig. 3 (b). From the contour slices as well the 3-D plot small deviations are observed which are due to the air gap between the retroreflective tape and the diaphragm.

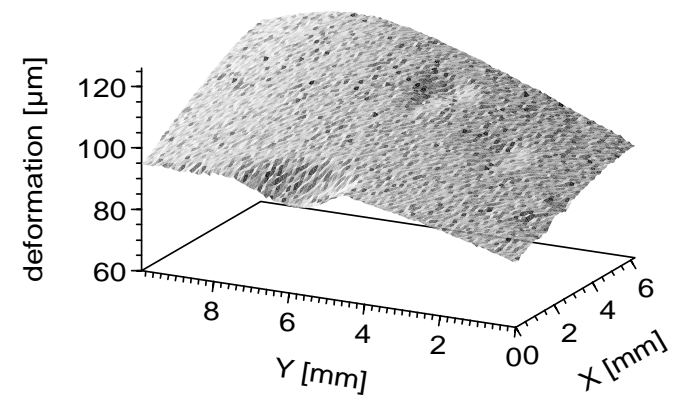

Fig. 3a: A circular diaphragm clamped along the edges was loaded at the center by $126 \mu \mathrm{m}$. and the 3-D plot of the deformation of the diaphragm. is shown

In order to make a comparative study of the three methods we investigated a crack in a thin metallic plate. The crack in the plate was created mechanically. The metallic plate was sprayed with a silver paint to increase the intensity of scattered light.

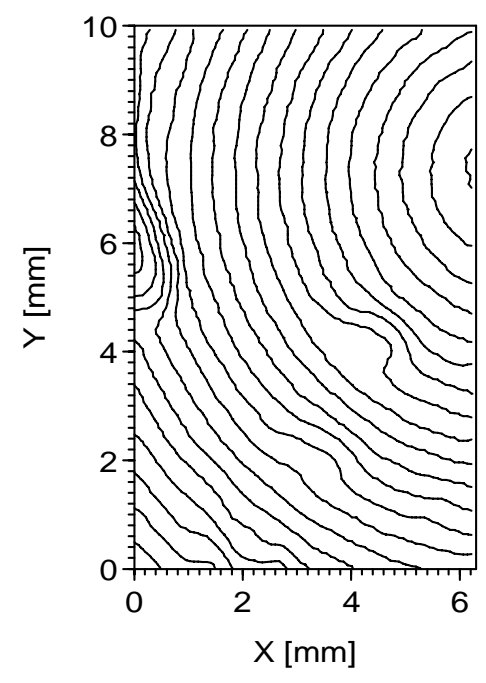

Fig. 3b: A circular diaphragm clamped along the edges was loaded at the center by $126 \mu \mathrm{m}$ and the contour plot of the deformation clearly showing the presence of a defect is shown. The defect was later determined to be the air gap between the retro-reflective tape and the diaphragm.

The point of loading was located for outof-plane and for slope measurement in a region close to the crack. For in-plane measurement the thin metallic plate was pulled from one end and clamped at the other. For the out-of-plane sensitive interferometer the object was deformed to $120 \mu \mathrm{m}$ and a region of $11 \times 7 \mathrm{~mm}^{2}$ around the crack was measured. Figures 4 (a) and (b) are the 3-D plots of the object deformation measured near the region of the crack. It can be seen that the crack is very prominent with this large deformation.

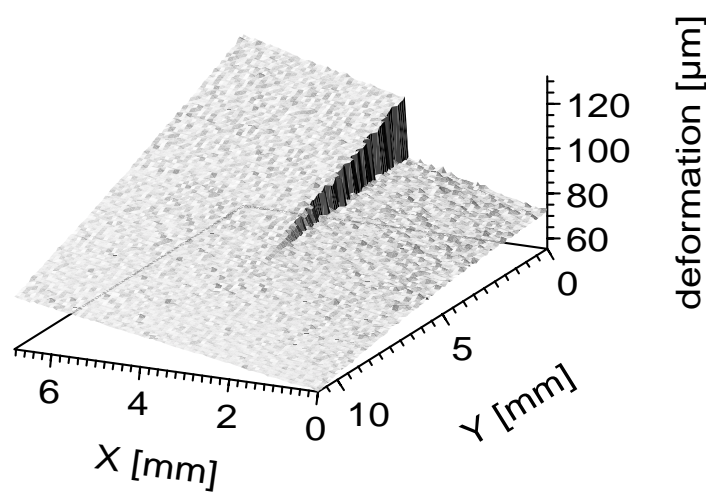

(a) 


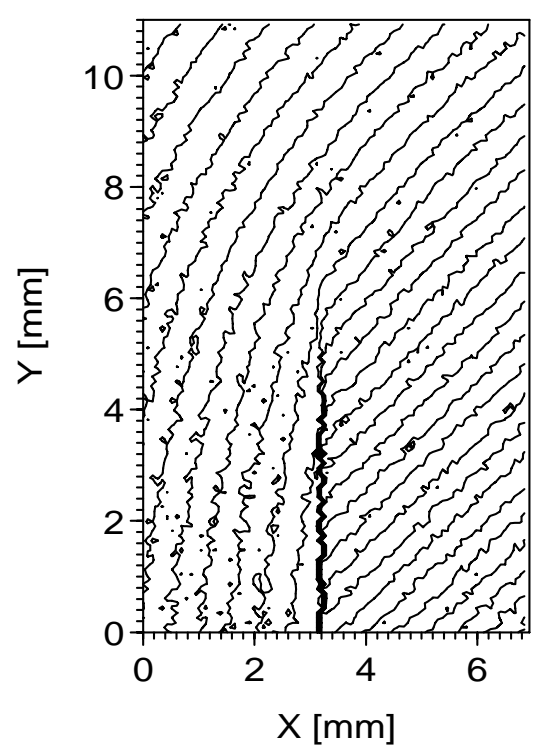

(b)

Fig. 4: Results for a rectangular plate clamped at the ends and loaded close to the region of the crack. The object was again deformed to $120 \mu \mathrm{m}$. a) shows the 3-D plot of the deformation around the region of the crack and b) shows the contour plots showing the deformation caused near the region of the crack.

\section{In-plane displacement:}

The experimental arrangement to measure in-plane displacement is shown in Fig. 5. An expanded and collimated laser beam is split into two beams of equal intensity by a beam splitter.

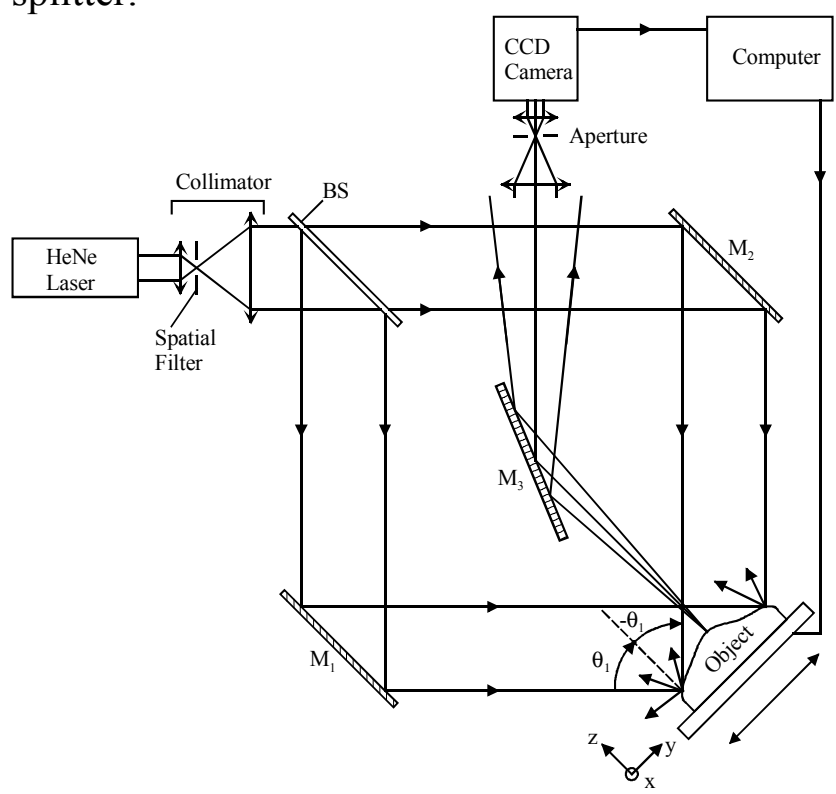

Fig. 5: Schematic diagram for the measurement of in-plane deformation.

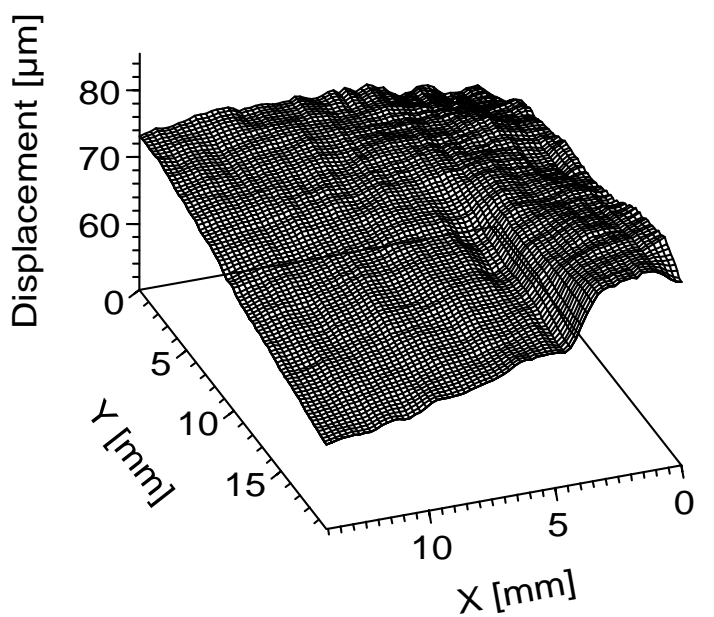

Fig. 6: The pseudo 3-D plot of the in-plane displacement measured near the region of the crack. The data was high pass filtered twice with a kernel of $5 \times 5$.

This beam is directed to illuminate an object from either side of its normal with $\theta_{1}$ degree and $-\theta_{1}$ degree via two mirrors $M_{1}$ and $M_{2}$. Using a telecentric system, the object is imaged onto the photo sensor of a CCD camera. In dual beam illumination speckle interferometry the light scattered from one beam serves as the reference for the light scattered by the other beam. This optical arrangement is used for measuring the ycomponent of the object displacement.

The rectangular thin metallic plate was clamped at both the ends. One clamped end was mounted on a computer controlled translation stage (MLTS) and the other end was clamped to the table. The end on the MLTS was displaced at a rate such that more than 100 cycles of modulation was obtained. A region of $20 \times 15$ $\mathrm{mm}^{2}$ of the object was investigated. Figures 6 shows the 3-D pseudo plot of the in-plane displacement measured near the region of the crack. The object at one end in this case was displaced by $80 \mu \mathrm{m}$. The crack is clearly visible in the plot where the end was displaced has more deformation. The drastic change in the in-plane displacement near the defect region can be noticed and can be used to identify crack.

\section{Slope measurement:}

The schematic of the Fourier-transform speckle pattern shearing interferometric method is shown in Fig. 7. Light from a laser is expanded and collimated, and a beam splitter 
$\mathrm{BS}_{1}$ is used to illuminate the object along the direction of its normal. The back scattered beam from the object is then directed to the photo sensor of the high speed CCD camera using a beam splitter $\mathrm{BS}_{2}$ and two mirrors $\mathrm{M}_{1}$ and $\mathrm{M}_{2}$.

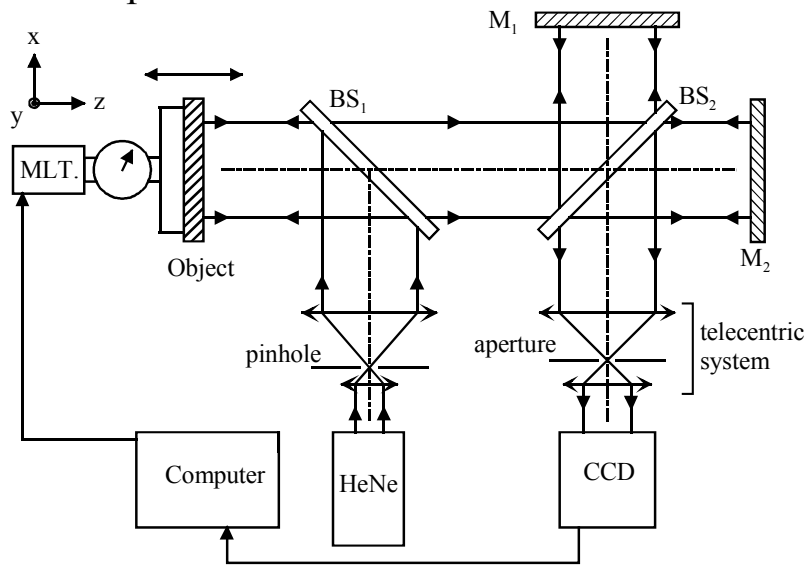

Fig. 7: Schematic arrangement of the speckle shear interferometric system to measure slope.

A telecentric system is used for imaging the object. Two images of the object are obtained from the two mirrors and they can be displaced in the image plane by tilting either one of them. Unlike in conventional interferometry or speckle interferometry where the object profile is compared to a plane reference beam, in speckle shearing interferometry a point in the object surface is compared to an adjacent point on the same object. Especially in our method of analysis, the relative temporal displacements of the two object points are compared. One of the mirrors was slightly tilted to displace one image along the $\mathrm{x}$-axis to generate desirable shear magnitude.

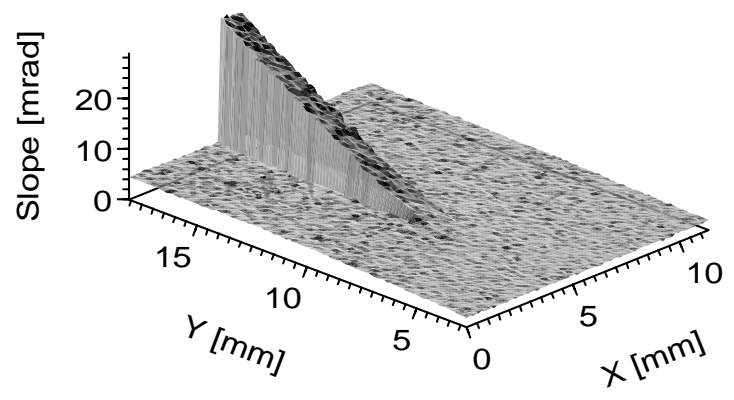

Fig. 8: The 3-D plot of the slope data measured near the region of the crack. The plate was deformed to around 500 $\mu \mathrm{m}$.

It is very well known that ESPSI is a useful tool for nondestructive testing (NDT).
However, the present method lends itself as a powerful combination of large deformation and easy data analysis. Therefore, small defects become visible under large object deformation. In our attempt we have deformed the object to around $1000 \mu \mathrm{m}$ in certain trials. Figures 8 show the 3-D plot of the slope measured for the plate. The shear at the object plane for trial was $1.0 \mathrm{~mm}$ and the object was deformed to $575 \mu \mathrm{m}$. In the region close to the crack, the slope is very large and is clearly observed from the plots. Integration of the slope data matches the displacement data shown in Fig. 5 (a).

\section{Comparison of the methods:}

Of the three methods proposed in this paper, the one least used conventionally is the dual beam illumination system. It should be mentioned that measurement of in-plane motion near the region of crack gives results equivalent to that of the other two methods. In shear interferometry as the slope changes are measured there is a minimum size of the defect that can be detected. This is dependent on the shear used in the system. In the method where fringe deviations are observed defect detection is sometimes too cumbersome when the general structure of the fringe pattern is not known. A prior knowledge is necessary to recognize deviations in fringe pattern. Further, in the present method of evaluation the accuracy of measurement for large deformation is high and enables easy detection of defect. Further, incremental displacement of the object deformation can also be extracted which provides information about the propagation of the crack or the development of defect in a region of observation. The most suitable method for defect detection with the three methods tried out is the out-of-plane sensitive interferometer.

\section{Conclusions:}

We have proposed a novel method of analysis in speckle interferometric method which can be used for NDT. Here object displacements in excess of $100 \mu \mathrm{m}$ have been recorded for out-of-plane and in-plane sensitive interferometers. A high speed CCD camera was used to acquire large number of frames of the 
object that is being deformed. In principle one can use a standard CCD camera to collect the data. The data is analyzed by Fourier-transform techniques where one of the side peaks is isolated by a band-pass filter and then is inverse Fourier transformed. The resultant phase oscillations are then unwrapped to obtain the total phase of the object deformation. Experimental results on studying crack with outof-plane, in-plane and slope sensitive interferometers suggest interesting applications for NDT analysis.

\section{Acknowledgments:}

We gratefully acknowledge the financial support by the 'German Research Association' DFG and the Alexander von Humboldt Foundation.

\section{References:}

1. K. Erf, Speckle Metrology, Academic Press, New York, 1978

2. R. Jones and C. Wykes, "Holographic and Speckle Interferometry," Cambridge University Press, London, 1983

3. R. S. Sirohi, "Speckle Metrology," Marcel Dekker, New York, 1993

4. C. Joenathan, "Speckle Photography, Shearography, and ESPI," in Optical Measurement Techniques and Applications, ed. P. K. Rastogi, Artech House Inc, Boston, London, 1997

5. C. Joenathan, B. Franze and H. J. Tiziani, "Oblique incidence and observation electronic speckle pattern interferometry," Applied Optics, 33, 7307-7311 (1994)
6. O. J. Lokberg, and O. Kwon, "Electronic speckle pattern interferometry using a $\mathrm{CO}_{2}$ laser," Opt. Laser Technol., 16, 187-192 (1984)

7. M. Takeda, H. Ina, and S. Kobayashi, "Fourier-transform method of fringe pattern analysis for computer-based topography and interferometry," J. Opt. Soc. Am, 72, 156160 (1982)

8. C. Joenathan, B. Franze, P. Haible, and H. J. Tizaini, "Speckle interferometry with temporal phase evaluation for measuring large object deformation," Accepted for publication in Applied Optics, 1997.

9. C. Joenathan, B. Franze, P. Haible, and H. J. Tizaini, "Large in-plane displacement measurement in dual beam speckle interferometry using temporal Fouriertransformation," Communicated to J. Modern Optics, 1997.

10. C. Joenathan, B. Franze, P. Haible, and H. J. Tiziani, "Novel temporal Fourier transform speckle pattern shearing interferometer," Communicated to Optical Engineering, 1997

11. H. J. Tiziani, B. Franze, and P. Haible, "Wavelength-shift speckle interferometry for absolute profilometry using a mode-hop free external cavity diode laser," J. Mod. Opt, 44, 1485-1496 (1997)

12. C. Joenathan, B. Franze, P. Haible, and H. J. Tizaini, "Shape measurement using temporal Fourier-transform in dual beam illumination speckle interferometry," Accepted for publication in Applied Optics, 1997. 